\title{
Min-Max Wireless Sensor Network Topology Optimization Localization Algorithm Based on RSSI
}

\author{
Qi Jin, Minhuan Huang, and Xiaohui Kuang
}

\begin{abstract}
Node localization technology is one of the key technologies in wireless sensor network. The traditional three edge localization algorithm is analyzed, which exists in poor stability in practical issues in wireless sensor networks. In this paper the Min-Max wireless sensor network topology optimization localization algorithm based on RSSI is proposed. Through the fixed anchor node coordinates using pretreatment method of the RSSI values, the accuracy of distance calculation is improved to obtain more precise positioning. And experiments using actual sensor nodes verify the effectiveness of the algorithm.
\end{abstract}

Index Terms-Wireless sensor network, RSSI, Min-Max localization algorithm.

\section{INTRODUCTION}

Wireless sensor network is deployed in the detection area by many micro-sensor nodes, to form a multi-hop selforganized network system through wireless communication. The purpose is to sense, collect and process network covering the perceived object region information and then send to the observer [1]. In wireless sensor network, location information of each sensor node is an indispensable part of nodes in the message, is one of the basic supporting technology for event position report, target tracking, geographic routing, network management, etc. Firstly, the sensor nodes must identify their location to specify "what happened in what position", positioning and tracking to achieve external goal. Secondly, the distribution of the master position sensor nodes, can improve the efficiency of routing network, realizes the automatic configuration load balancing and network topology of the network, to cover the whole quality improvement network. Therefore, the mechanism or algorithm must be obtained to achieve the positioning of each node in wireless sensor networks [2].

The simplest method of wireless sensor network localization is loading GPS for each node receiver to determine the location of the node. However, due to the cost, node energy constraints and GPS with certain requirements for the deployment of environmental constraints, this method leads to low feasibility scheme. Therefore, only a few nodes by GPS or the previously deployed in specific locations way obtain their own coordinate. In addition, node localization in wireless sensor network involves many aspects, including positioning accuracy, scale of the network, anchor node density, network fault tolerance and robustness as well as the power consumption, how to balance the

Manuscript received May 19, 2014; revised August 14, 2014.

The authors are with the National Key Laboratory of Science and Technology on Information System Security, Beijing, China (e-mail: kingkey@sohu.com,

xiaohui_kuang@163.com). various relations for the positioning problem of wireless sensor networks is challenging.

In Section II, the principle of the three edge localization algorithm is briefly introduced. In Section III, the Min-Max algorithm is described. In Section IV, the RSSI range loss calculation method of Min-Max localization algorithm is optimized. And the effectiveness of this proposed method is verified through the experiment in an actual wireless sensor network in Section V. Conclusions are presented in Section VI.

\section{THREE EDGE LOCALIZATION ALGORITHM}

According to positioning system, localization algorithms of wireless sensor network are divided into two categories [3]: range-based localization and range-free localization. The former obtains more accurate positioning results, which produces a large amount of calculation and communication overhead. The latter only relies on network connectivity information of node positioning with low energy consumption. The basic method to calculate the node position comprises three edge localization method, and maximum likelihood estimation method. At present, ranging techniques mainly include TOA (time of arrival), TDOA (time difference of arrival), AOA (angle of arrival) and RSSI (received signal strength indicator) [4]-[15]. Compared with the single range-free algorithm, RSSI with more useful information and less hardware resources, can improve the positioning accuracy. Meanwhile, due to no need to measure absolute distance or location between nodes, which deduces the requirements of hardware, the cost of nodes is more suitable for large-scale sensor networks.

The basic principle of three edge localization algorithm is used three beacon nodes as the center of the three circles, and the radius is equal to the distance from the three beacon nodes to the unknown node respectively. The coordinates of unknown node is calculated from the intersection of the three circles in the plane, which is shown in Fig. 1. A, B, and $\mathrm{C}$ node is the anchor node coordinates, respectively (XA, $\mathrm{YA}),(\mathrm{XB}, \mathrm{YB})$, and (XC, YC). D is the unknown node to be measured, coordinates (XD, YD). If the system obtains the RSSI values from each anchor node to the unknown node, namely $\mathrm{D}$ to $\mathrm{A}, \mathrm{B}, \mathrm{C}$ distance of $\mathrm{rA}, \mathrm{rB}, \mathrm{rC}$ can be computed through the loss model of RSSI and distance.

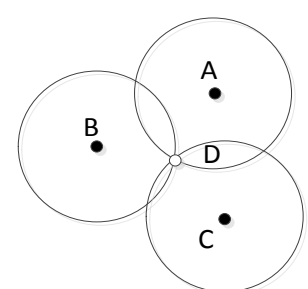

Fig. 1. Three edge localization algorithm geometrical configuration. 
According to Fig. 1, the relationship can be given as:

$$
\begin{aligned}
& \left(X_{A}-X_{D}\right)^{2}+\left(Y_{A}-Y_{D}\right)^{2}=r_{A}^{2} \\
& \left(X_{B}-X_{D}\right)^{2}+\left(Y_{B}-Y_{D}\right)^{2}=r_{B}^{2} \\
& \left(X_{C}-X_{D}\right)^{2}+\left(Y_{C}-Y_{D}\right)^{2}=r_{C}^{2}
\end{aligned}
$$

The D node can be calculated as:

$$
\left[\begin{array}{l}
X \\
Y
\end{array}\right]=\left[\begin{array}{l}
2\left(X_{A}-X_{C}\right) 2\left(Y_{A}-Y_{C}\right) \\
2\left(X_{B}-X_{C}\right) 2\left(Y_{B}-Y_{C}\right)
\end{array}\right]^{-1}\left[\begin{array}{l}
X_{A}^{2}-X_{C}^{2}+Y_{A}^{2}-Y_{C}^{2}+r_{C}^{2}-r_{A}^{2} \\
X_{B}^{2}-X_{C}^{2}+Y_{B}^{2}-Y_{C}^{2}+r_{C}^{2}-r_{B}^{2}
\end{array}\right]
$$

But in actual process, the algorithm error of RSSI measuring and reflection and scattering of the wireless signal in the transmission may occur, which will cause the three circles may not be at a point, or even the two circles may not intersect, as shown in Fig. 2.

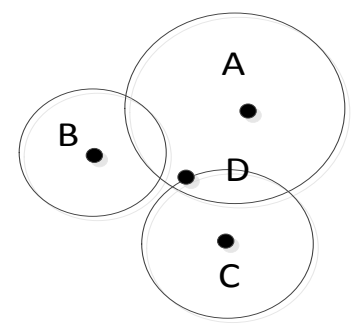

Fig. 2. The distortion of localization algorithm.

In this case, the error of three edge localization algorithm will increase, which affects the stability of localization algorithm in practical application.

\section{MIN-MAX LOCALIZATION ALGORITHM AND CALIBRATION}

Min-Max localization algorithm is one of the most widely applied algorithms application, which has a high accuracy, easy calculation and good scalability. The Min-Max algorithm is similar to three edge localization algorithm. The distance di from the anchor node to the measured node is calculated by using the transmission distance loss model. Setting the anchor node is the center of the circle, and the radius of the circle is equal to di. And further the external square of the circular area can be obtained, which is a $2 \mathrm{D}$ square location point, as shown in Fig. 3.

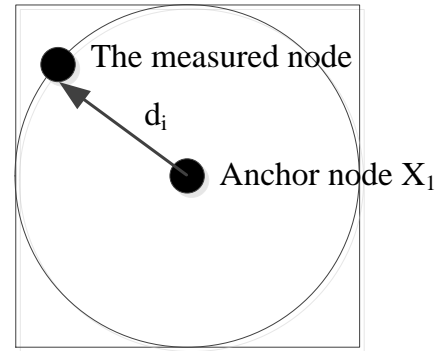

Fig. 3. 2D square area of the located node.

Positioning system usually has more than two anchor nodes, which lead to at least two 2D square areas. Then the measured node is in the public region of two square areas, which is shown in Fig. 4.

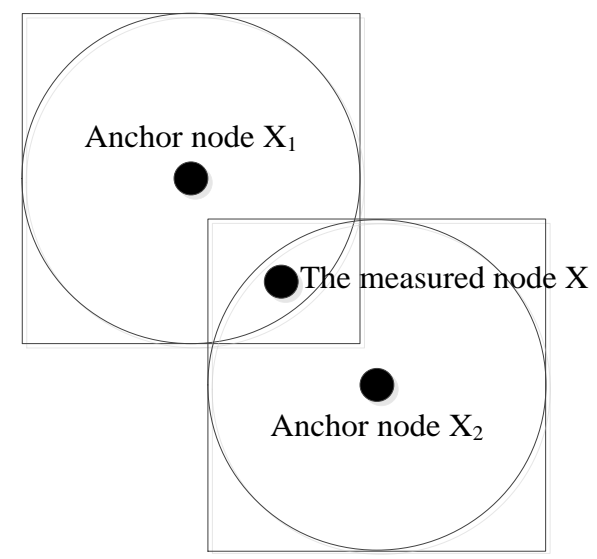

Fig. 4. Min-Max algorithm diagram.

The public region can be calculated as follows:

$\left(\max \left(X_{i}-d_{i}\right), \max \left(Y_{i}-d_{i}\right)\right) \times\left(\min \left(X_{i}+d_{i}\right), \min \left(Y_{i}+d_{i}\right)\right)$

In Min-Max localization algorithm, the more square the anchor node contains, the smaller the public square area is, and estimation is more accurate. The process of Min-Max algorithm is not related to the circle itself. Compared to the three edge localization algorithm, it is more convenient for calculation. According to the public area formula, the centroid of public area can be obtain by using the centroid method to get the estimated coordinates of $X$, which can be calculated as follows.

$\left(\frac{\left(\max \left(X_{i}-d_{i}\right)+\left(\min \left(X_{i}+d_{i}\right)\right.\right.}{2}, \frac{\max \left(Y_{i}-d_{i}\right)+\min \left(Y_{i}+d_{i}\right)}{2}\right)$

\section{RSSI LOCALIZATION ALGORITHM CALIBRATION}

The principle of RSSI ranging technology is the conversion loss model of the wireless signal in the transmission rule into the distance. The relation between distance and RSSI can be expressed as follows.

$$
d=10^{A N S(R S S O)-A} /(10 \times n)
$$

The optimum value for $\mathrm{A}$ is in the range of $45-49$, and for $n$ is 3.25-4.5. Four anchor nodes are deployed at different positions to obtain the distance from each anchor node to target node, and upload to the sink node. As shown in Fig. 5, four anchor nodes are A, B, C and D.

Due to the difference of each node itself, the model parameters are set not completely same, otherwise it will lead to a certain error. Therefore, it is necessary to separate calibration for each anchor node. According to Eq. (5), the RSSI values ( $A$ and $n$ ) of corresponding distance can be calculated by MATLAB.

In order to avoid the four anchor nodes access to information upload at the same time, which will lead to the sink node function exhaustion, anchor nodes are equipped with different delay upload time. The sink node $\mathrm{S}$ will set the distance information and the location information of anchor nodes as the known conditions, by using Min-Max localization algorithm to calculate the coordinates of the relative position of the target node, which is shown in Fig. 5. 


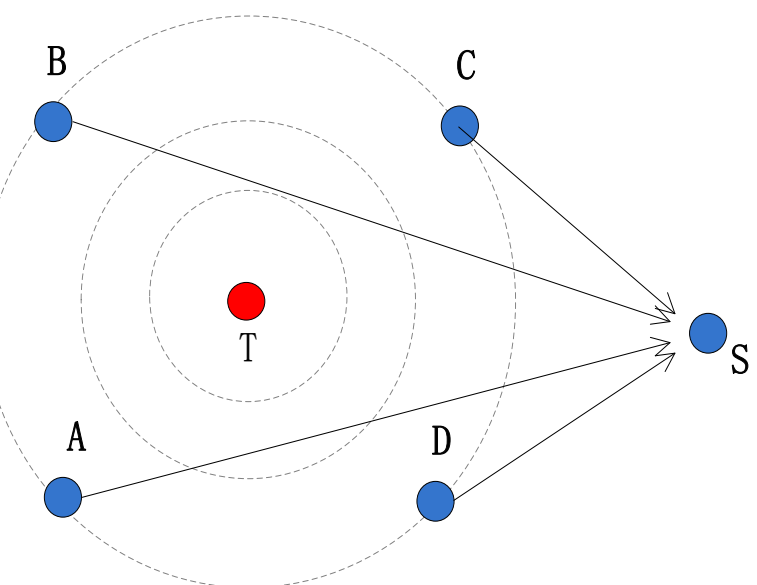

Fig. 5. 2D square area of the located node.

The relationship between RSSI and distance is obtained through experiments. RSSI is measured at different distance. Each group of test 50 chooses one of the stable value to get simulation curve to determine the parameters of transformation model of RSSI and the distance values, which is shown in Fig. 6.

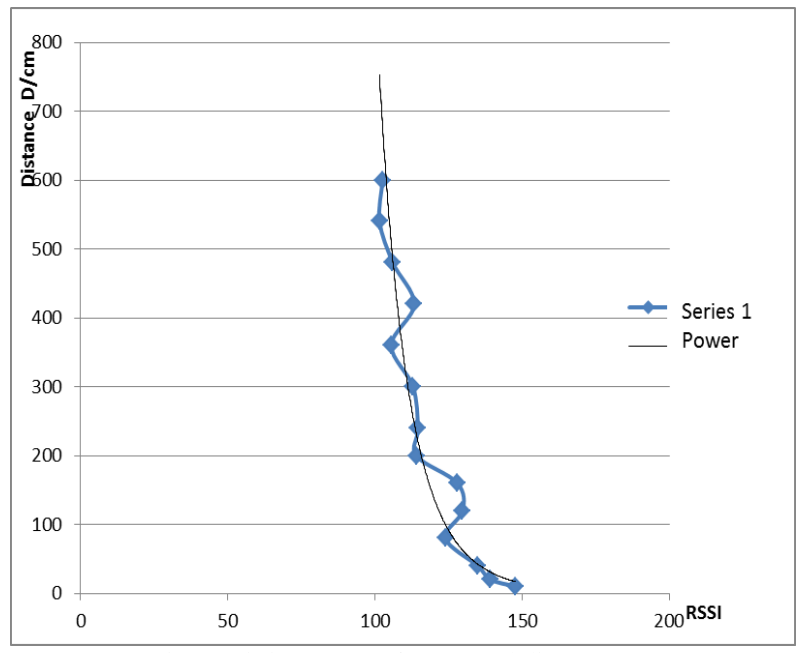

Fig. 6. Fitting curve of RSSI and distance.

The steps of this localization algorithm can be described as follows:

- Calibrate anchor node RSSI module;

- Perception of the target node location information by anchor nodes;

- Gather node receiving the information of anchor nodes;

- Calculate node positioning.

Through the calibration of the anchor node RSSI module and improving the gathering node positioning algorithm, the positioning precision can reach to 3 meters.

\section{EXPERIMENTAL VERIFICATION}

Physical layout in experiment is shown in Fig. 7. Among them, the node type and address are labeled beside the node.

Taking an example of node $15 \mathrm{CD}$, which is shown in Fig. 7, the position of node in the topology before positioning is shown in the triangle mark, locating in the upper left of another target node $00 \mathrm{CD}$ in Fig. 8, and the actual location relations are not consistent in Fig. 7. Location information is shown in red box in Fig. 8.

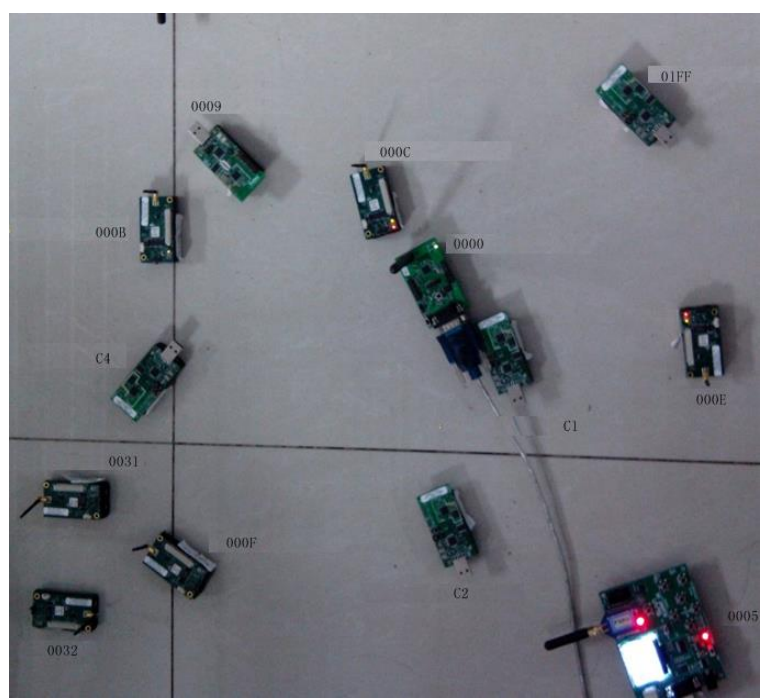

Fig. 7. Topology detection reference scenario.

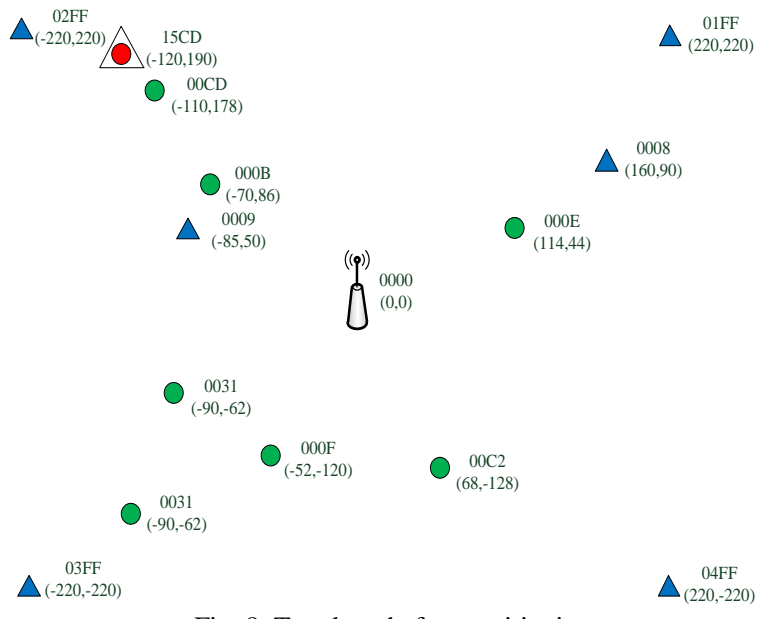

Fig. 8. Topology before positioning.

The topology after positioning is shown in Fig. 9. The position of node $15 \mathrm{CD}$ is revised, which is inside the triangle, locates in the bottom left of node $00 \mathrm{CD}$, and corresponds to Fig. 7. Positioning technology improves the topology detection target reduction ability to a certain extent.

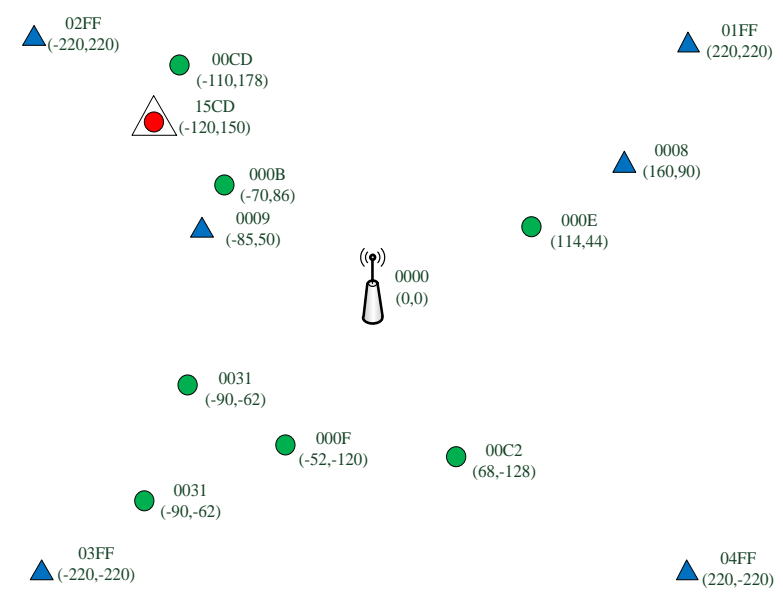

Fig. 9. Topology after positioning.

\section{CONCLUSIONS}

This paper is focus on the positioning technology of RSSI. In Min-Max localization algorithm, by using RSSI range loss calculation method, optimizes the anchor node location 
information, and obtains the optimization of Min-Max localization algorithm. This algorithm does not need additional equipment, and through experimental results with actual sensor nodes, the optimization Min-Max algorithm can improve the accuracy of distance calculation and obtain more precise positioning.

\section{REFERENCES}

[1] C. Karlof and D. Wagner, "Secure routing in wireless sensor networks: Attacks and countermeasures," AdHoc Networks Journal, vol. 1, no. 2-3, pp. 293-315, September 2003.

[2] Y. Q. Ma, "Research and implementation of positioning technology based on wireless sensor network," Beijing University of Posts and Telecommunications \& Technology, 2006, pp. 23-24.

[3] V. V. Challas et al., "Nonmetric MDS for sensor localization," in Proc. the 3rd International Symposium on Wireless Pervasive Computing, 2008, pp. 396-400.

[4] K. Langendoe and N. Rehers, "Distributed localizmion in wireless sensor networks: a quantitative comparison," Computer Networks, vol 43, no. 4, pp. 499-518, 2003.

[5] B. F. Maog and B. Anderson, "Wireless sensor network localization techniques," Computer Networks, vol. 51, no. 10, pp. 2529-2553, 2007.

[6] X. Sheng and Y. H. Hu, "Maximum likelihood multiple-source localization using acoustic energy measurements with wirelesssensor networks," IEEE Transactions on Signal Processing, vol. 53, no. 1, pp. 44-53, 2005.

[7] O. Ssuk and Jiauhc, "Localization withmobile anchor point $s$ in wireless sensor networks," IEEE Transactions on Vehicular Technology, vol. 54, no. 3, pp. 1187-1197, 2005.

[8] G. Aloor and L. Jacob, "Distributed wireless sensor network localization using stochastic proximity embedding," Computer Communications, vol. 33, no. 6, pp. 745-755, 2010.

[9] J. Lee, W. Chung, and E. Kim, "A new range-free localization method using quadratic programming," Computer Communications, vol. 34, no. 8, pp. 998-1010, 2010.

[10] C. Li, Y. Li, Y. Shen, L. Liu, and Q. Cao, "An optimization algorithm for wireless sensor networks localization using multiplier method," in Proc. the 3rd International Joint Conference on Computational Science and Optimization, Huangshan, Anhui, China, 2010, pp. 337341.

[11] L. Liu and E. Manli, "Localization for wireless sensor networks by combining TFDA and FMCW," in Proc. the IEEE International Conference on Mechatronics and Automation, Xian, China, 2010, pp. 945-950.

[12] P. Motter, R. Allgayer, I. Müller, and E. de Freitas, "Practical issues in wireless sensor network localization systems using received signal strength indication," in Proc. the Sensors Applications Symposium, San Antonio, TX, USA, 2011, pp. 227-232.

[13] M. Sayadnavard, A. Haghighat, and M. Abdechiri, "Wireless sensor network localization using imperialist competitive algorithm," in Proc. the 3rd IEEE International Conference on Computer Science and Information Technology, Chengdu, China, 2010, vol. 9, pp. 818-822.

[14] S. Shekofteh, M. Yaghmaee, M. Khalkhali, and H. Deldari, "Localization in wireless sensor networks using tabu search and simulated annealing," in Proc. the 2nd International Conference on Computer and Automation Engineering, Singapore, 2010, pp. 752757.

[15] Q. Shi, C. He, H. Chen, and L. Jiang, "Distributed wireless sensor network localization via sequential greedy optimization algorithm," IEEE Transactions on Signal Processing, vol. 58, no. 6, pp. 3328$3340,2010$.

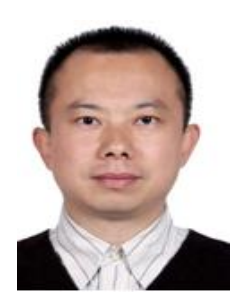

Qi Jin was born in 1975, he is an associate professor now with National Key Laboratory of Science and Technology on Information System Security, Beijing, China. His major research interests include wireless sensor network, and the internet of things.

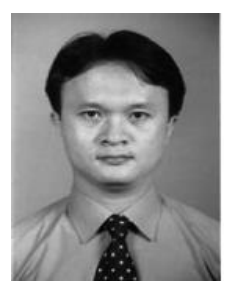

Minhuan Huang was born in 1971, he is working as a professor now with National Key Laboratory of Science and Technology on Information System Security, Beijing, China. His major research interests include computer networks, computer security, and software architecture.

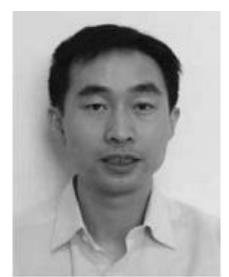

Xiaohui Kuang was born in 1975 , he is working as an associate professor now with National Key Laboratory of Science and Technology on Information System Security, Beijing, China. Hi major research interests include wireless network, and information security. 